\title{
Pierre Moessinger, Voir la société. Le micro et le macro
}

Paris, Hermann, 2008

\section{David Hoff}

\section{(2) OpenEdition}

\section{Journals}

Édition électronique

URL : http://journals.openedition.org/questionsdecommunication/1079

DOI : 10.4000/questionsdecommunication. 1079

ISSN : 2259-8901

\section{Éditeur}

Presses universitaires de Lorraine

\section{Édition imprimée}

Date de publication : 1 juillet 2009

Pagination : 475-477

ISBN : 978-2-86480-989-0

ISSN : 1633-5961

\section{Référence électronique}

David Hoff, «Pierre Moessinger, Voir la société. Le micro et le macro», Questions de communication [En ligne], 15 | 2009, mis en ligne le 18 janvier 2012, consulté le 22 septembre 2020. URL : http:// journals.openedition.org/questionsdecommunication/1079 ; DOl : https://doi.org/10.4000/ questionsdecommunication.1079

Ce document a été généré automatiquement le 22 septembre 2020.

Tous droits réservés 


\section{Pierre Moessinger, Voir la société. Le micro et le macro}

Paris, Hermann, 2008

David Hoff

\section{RÉFÉRENCE}

Pierre Moessinger, Voir la société. Le micro et le macro. Paris, Hermann, coll. Société et pensées, 2008, $260 \mathrm{p}$.

1 Analyser un phénomène social, c'est le voir avant de le concevoir. C'est ce que tente de démontrer Pierre Moessinger - professeur de sociologie à l'université de Genève dans cet ouvrage. En alternant analogies suggestives et réflexion épistémologique, ce dernier tente de mettre en lumière la question du regard en sciences humaines et sociales, notamment en psychologie et en sociologie.

2 Le premier chapitre - «De près ou de loin, et autres questions fondamentales » (pp.19-98) - commence par une métaphore décrivant un atterrissage. L'auteur procède à un «zoom » progressif, partant de 10000 mètres d'altitude pour arriver au sol en décrivant ce qu'il voit. Par cette illustration, il tente de faire comprendre que la distance joue un rôle primordial dans ce qu'il distingue. Proche des choses, on voit les micro-propriétés, "en s'en éloignant, on voit davantage de choses, mais surtout, apparaissent les relations entre les choses, leur organisation ou leur "structure " perceptive » (p. 32). Ce que nous voyons serait-il alors fonction de notre point de vue? C'est une des questions posées dans cet ouvrage à laquelle il tente de répondre. Inspiré par jean Piaget qui considère la perception comme une construction, l'auteur donne une première réponse en affirmant que celle-ci n'est indépendante ni de l'expérience, ni de la cognition : « Il faut bien concevoir l'espace pour bien voir » (p. 36).

3 Revenant sur la problématique des niveaux, l'auteur rejette à la fois les approches relevant d'un «individualisme » ou d'un « holisme radical », parodiant ces approches par une description allégorique, volontairement absurde, d'extra-terrestres qui 
observeraient notre société afin de démontrer que sa vision " émergentiste » s'oppose à ces deux approches qui amènent à « des conceptions épistémologiques ou ontologiques à éviter » (p. 80),caractérisées par la banalité et la subjectivité ou l'hyper-rationnalisme et l'hyper-empirisme.

Pierre Moessinger consacre le deuxième chapitre-«Comme des fils invisibles 》 (pp. 99-126) -à une description de passants dans la rue, suivie d'une réflexion sur la meilleure manière de restituer les phénomènes observés, puis essaye de comprendre ce qui assure la coordination des personnes dans ce flux. Distinguant cette foule de passants d'un orchestre, le chercheur tente de différencier le réseau du système. Dans le degré de cohésion, dans la manière de se coordonner ainsi que dans la densité de connexion, il repère autant de caractéristiques qui les séparent. Contrairement au réseau, le système crée une connexion "assez forte pour «tenir » le tout, ou pour permettre son intégration»( approches systémiques qui, selon lui, font l'amalgame de ces deux manières d'être relié aux autres. L'auteur distingue la catégorie de ces deux notions, du fait que la première ne relève d'aucune connexion.

Dans un troisième chapitre - "Les dynamiques sociales » (pp. 127-200) -, c'est la notion de dynamique sociale qui est interrogée. Ce mécanisme se met en place lors de la création d'un système et avant son « désassemblage ». À travers des exemples comme l'adoption du clavier QWERTY, l'heure d'arrivée d'étudiants à des cours ou le succès d'une pizzeria ou d'un film, il évoque les différentes approches de la dynamique sociale. Selon les approches, cette dernière peut être réduite à une maximisation, considérée comme un phénomène où jouent initiation et hasard, ou bien être uniquement appréhendée à l'aide de l'analyse du rôle des initiateurs. L'explication de cette notion est ensuite soumise aux modèles d'analyse économiques. Et la conclusion de ce chapitre débouche sur l'évocation puis la critique du modèle de Kirmann qui explique les changements de situation en faisant intervenir deux variables principales: la probabilité d'être converti et celle de changer d'état de manière spontanée. Pour Pierre Moessinger, ce modèle a pour caractéristique d'être en décalage avec la réalité, mais par son "infidélité féconde [il] per- met de comprendre ce que le réel n'est pas » (p. 200). L'auteur s'intéresse aussi aux caractéristiques qui structurent l'interaction. Pour lui, quatre facteurs constituent les piliers de l'interaction: les conduites interindividuelles sont cadrées par des règles et des normes, des rapports de force, des contraintes de l'environnement ou de la situation. Enfin, l'interaction est expliquée par une capacité de décision libre accordée à l'individu.

Les phénomènes sociaux ainsi que leur rapport à la totalité sont abordés dans un dernier chapitre - «Emergence et fonctionnement de la totalité », (pp. 201-242). Il y a phénomène social dès lors qu'il existe " des coordinations psycho-motrices et sociomotrices et via ces coordinations motrices, des coordinations mentales [qui sont à la base d'un système social] et d'un changement de l'état du système»(p. 202). Le phénomène social est donc perçu dans une perspective dynamique, tant dans la conception de l'interaction que dans celle du changement dans un système. L'auteur précise la limite qu'il donne à la notion. Il n'entend par système social que ceux qui sont réels. Il les oppose aux sémantiques et conceptuels qui, selon lui, ne sont pas de véritables systèmes sociaux. Il aborde ensuite la notion d'institution en sociologie et en psychologie. Dans la première discipline, il reproche aux chercheurs de confondre cette notion avec celle d'organisation. Selon le chercheur, Émile Durkheim avait tendance à 
réifier l'organisation, mais au fil du temps, la sociologie aurait progressé en la matière. Il reproche ensuite aux psychologues d'être aveugles à cette notion. Ceci viendrait de ce que la psychologie conçoit les individus comme étant "guidés de l'intérieur ou contrôlés de l'extérieur» (p. 209), avec, plus particulièrement pour la psychologie sociale, une dissimulation du facteur institutionnel. La prise de conscience de ce handicap conduirait à relativiser nombre d'études menées dans cette discipline en diminuant considérablement la représentativité des données recueillies et la portée des analyses. Enfin, Pierre Moessinger pose les bases d'une sociologie de la totalité qui expliquerait les conduites en fonction des quatre facteurs cités plus haut et qui mettrait l'accent sur l'émergence. La définition de l'émergence est reprise à Mario Bunge qui l'entend comme une propriété intrinsèquement absente des composantes d'un système. Ce dernier voit chez Émile Durkheim, «qui avait compris que les individus se conduisent autrement dans des systèmes sociaux qu'à l'état isolé » (p. 229), une tentative de saisir le concept d'émergence et de micro-émergence qui a malheureusement été empêchée par une trop grande insistance sur la contrainte. Pour l'auteur, la micro-émergence est le changement de propriétés chez l'individu et l'apparition de nouvelles caractéristiques induites par un changement d'état au niveau macro. Il conclut que l'explication d'un phénomène social est un juste milieu à trouver entre les deux niveaux, macro et micro, qu'elle doit porter autant sur les systèmes que sur les intentions et les processus qui relient le système à ses parties ou à son environnement. Cette explication par les processus ne doit être ni causale ni téléologique : elle doit contribuer à enrichir la complexité de la connaissance, «la nouveauté ne s'en va pas quand on en propose une explication, mais le mystère s'en va quand on cherche à l'expliciter » (p. 241).

7 Le principal intérêt de l'ouvrage est de penser la notion de distance et de relation entre les niveaux. L'auteur propose une réflexion de portée générale et une posture originale. Il emprunte à plusieurs disciplines des éléments de théorie qu'il mobilise dans la construction de concepts complexes et dynamiques mais qui peuvent se révéler abstraits et, pour certains, peu opératoires. Dans un livre consacré à une réflexion ontologique et épistémologique, on peut regretter de trouver un état des lieux trop superficiel et tranché des points de vue relatifs aux différentes disciplines constituant les sciences sociales, renvoyant hâtivement la psychologie vers une approche systémique et assignant à la sociologie une vision dogmatique et herméneutique. On peut aussi reprocher à Pierre Moessinger de penser l'émergence et la micro-émergence comme des parties d'un même processus, à savoir l'action de la partie sur le tout ou l'effet d'un élément extérieur sur un système répercuté vers ses membres, laissant peu de marge à la prise en compte de la liberté individuelle dans l'explication de l'action. Reproche qu'il adresse à maintes reprises aux approches systémiques, tout comme le fait de ne pas laisser à l'individu la possibilité d'être lui- même générateur de changement. Malgré ces limites, la vision complexe et transdisciplinaire que propose Pierre Moessinger conduit le lecteur à réfléchir sur l'importance du regard que luimême porte sur un monde social riche et à lui faire prendre conscience que l'angle de vue et l'échelle conditionnent la sélection des phénomènes qu'il analyse. Voir, c'est finalement mettre en lumière des éléments constituant un monde social riche, simplifier la complexité des mécanismes sociaux qui les ordonnent et les coordonnent. C'est aussi se situer sur un continuum reliant l'interaction élémentaire à la macrostructure. 


\section{AUTEURS}

DAVID HOFF

CREM, université Paul Verlaine-Metz

davidhoff@laposte.net 